\title{
Towards Machine Vision-enabled STEM EELS for High-throughput Quantification of Grain Boundary Electronic Structure
}

Sai Muktevi ${ }^{1}$, David Carreon ${ }^{2}$ and William Bowman ${ }^{3}$

${ }^{1}$ Dept. of Computer Engineering, University of California, Irvine, Irvine, California, United States, ${ }^{2}$ Dept. of Computer Science, University of California, Irvine, Irvine, California, United States, ${ }^{3}$ University of California, Irvine, Irvine, California, United States

Most functional and structural materials are polycrystalline, made of a large number of single crystalline grains aggregated together. Grain boundaries (GBs) - the interfaces between differently oriented grains of polycrystals - strongly influence, if not determine, many functional, mechanical and physical properties of the materials $[1,2]$. Understanding the diversity in electronic structure of the GBs in a polycrystalline sample thus enables one to design materials with predictable and favorable properties.

In this contribution, I will discuss our progress towards developing and employing a new software for autonomous imaging and spectroscopic analysis of GBs by electron energy-loss spectroscopy (EELS) in the STEM. This high-throughput approach will help build the largest experimental dataset on GB electronic structure in a polycrystalline material, giving access to local information from the many unique GBs in a specimen. Applying a high-throughput STEM EELS data acquisition scheme capable of probing large numbers of unique GBs will reveal previously-inaccessible statistical insights about electronic structures within the GB population.

This will be aided by an extensive manual data acquisition effort carried out using a test specimen, which will serve as a benchmark against which we can compare the autonomously-gathered GB data. Our preliminary work has resulted in basic software control over the STEM EELS data acquisition process, and are continuing work towards a live proof-of-concept demonstration on test specimens. The software we have built so far is capable of ingesting live video streams generated by any electron detector in the STEM, processing the video frames to find loci of various features such as GBs, e.g. Figure 1, and performing EELS line profile data acquisitions. A comparable system for nanoparticle tracking and analysis by scanning transmission electron microscope (STEM) was recently reported [3], though specific domain knowledge is needed to develop a system able to accurately and reliably detect, track and analyze GBs autonomously.

Figure 1. GBs in a STEM dark field image (a) of a polycrystalline oxide can be detected (b) using edgefinding algorithms, and their rotational orientation (c) can be determined automatically. We are now integrating this software into a STEM spectroscopic data acquisition system for high-throughput GB analysis.
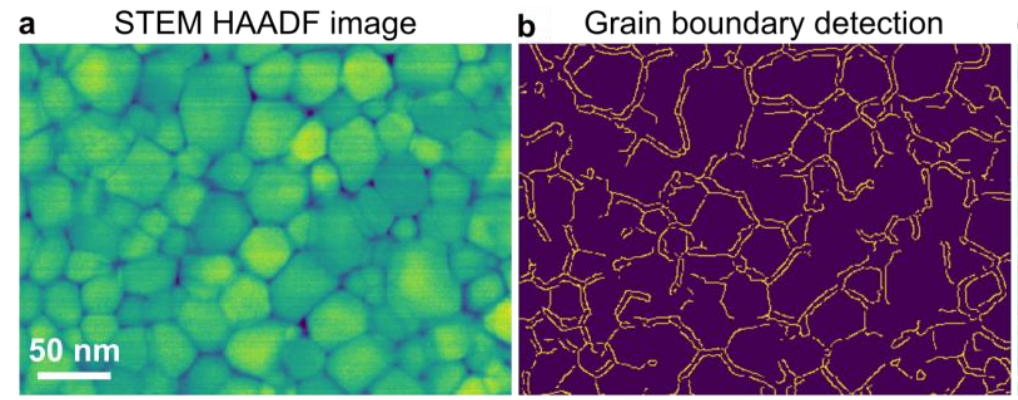

c Grain boundary orientations 
Figure 1. GBs in a STEM dark field image (a) of a polycrystalline oxide can be detected (b) using edgefinding algorithms, and their rotational orientation (c) can be determined automatically. We are now integrating this software into a STEM spectroscopic data acquisition system for high-throughput GB analysis.

\section{References}

1. Bowman WJ, Kelly MN, Rohrer GS, Hernandez CA, Crozier PA (2017) Enhanced ionic conductivity in electroceramics by nanoscale enrichment of grain boundaries with high solute concentration. Nanoscale, 9:17293-17302. https://doi.org/10.1039/C7NR06941C

2. Bowman WJ, Darbal A, Crozier PA (2019) Linking Macroscopic and Nanoscopic Ionic Conductivity: A Semiempirical Framework for Characterizing Grain Boundary Conductivity in Polycrystalline Ceramics. ACS Applied Materials \& Interfaces, https://doi.org/10.1021/acsami.9b15933

3. Uusimaeki T, Wagner T, Lipinski H-G, Kaegi R (2019) AutoEM: a software for automated acquisition and analysis of nanoparticles. Journal of Nanoparticle Research, 21(6):122. https://doi.org/10.1007/s11051-019-4555-9

This work was funded by a UCI new faculty start-up grant, and performed at the Irvine Materials Research Institute (IMRI, https://imri.uci.edu/) 